Original Research Paper

\title{
Utilizing of Big Data and Predictive Analytics Capability in Crisis Management
}

\author{
Mohammad Atwah AL-Ma'aitah \\ Department of MIS, AL-Balqa' Applied University, Jordan
}

Article history

Received: 02-12-2019

Revised: $17-01-2020$

Accepted: 07-03-2020

Email: dr-moh1975@bau.edu.jo

\begin{abstract}
This study investigates the impact of Big Data and Predictive Analytics (BDPA) capability on crisis management in the Greater Amman Municipality (GAM), Jordan. Effective utilization of BDPA is considered in the study to depend upon the five dimensions: Tangible resources, technical skills, management skills, organizational learning and data driven culture. Crisis management is classified into the three stages of crisis preparedness, crisis response and crisis recovery. A32 item questionnaire was developed for the purpose of the study, which was completed by 140 participants; with128 responses deemed suitable for research analysis. PLS2 software was used to analyze the data. The study results confirm that the Greater Amman Municipality has medium levels of big data availability and predictive analytics capability overall. The study's findings further demonstrate that big data and predictive analytics capability has a significant impact on crisis management. The main recommendation of the study is that organizations enhance their ability to utilize big data through expanding their tangible resources infrastructure, including machine learning algorithms, python software, simulation and regression analysis for understanding huge volumes of data. In addition, the study recommends that organizations improve the technical skills of their staff by providing the necessary training for performing big data analysis.
\end{abstract}

Keywords: Big Data, Predictive Analytics, Crisis Preparedness, Crisis Response, Crisis Recovery

\section{Introduction}

Crisis management and disaster control are among the top priorities for organizations worldwide. However, despite the attention and primacy organizations give this issue, effective crisis management remains difficult to achieve (Besaleva and Weaver, 2016). Organizational disasters can cause widespread environmental and humanitarian harm in addition to devastating company finances and reputation (Mitroff et al., 1987). Today's uncertainty and market unpredictability were increased in the economic environment. Many countries and organizations have faced repercussions of the contemporary global financial crises and most struggled to manage their financial resources efficiently in face of those (Zeitun and Benjelloun, 2013). Changes in the technological environment and constant pressures have pushed organizations to adopt various approaches to managing inevitable crises and disasters. In a world of constant technological updates, organizations must continuously upgrade these methods to reduce the risk of facing a crisis they cannot address (Coombs, 2011). Information Technology (IT) has become one of the most important tools for forecasting and managing unexpected organizational challenges. Most contemporary organizations rely on information technology to collect, process, store, transmit and retrieve information. Research has indicated that increased IT capabilities can enhance organizational ability to exploit opportunities and avoid threats ( $\mathrm{Lu}$ and Ramamurthy, 2011). The increasing volume of information that organizations possess, due in large part to the increase in modern technologies (such as smart devices, scanning devices, cloud computing, Internet of Things, etc.,) that explode the speed and extent to which organizations can obtain information, has made the process of handling and analyzing data very complex (Wamba et al., 2015). Accordingly, multiple new techniques have appeared allowing organizations to utilize their overwhelming amount of data. The most 
relevant of these and the one we will focus on here, is Big Data and Predictive Analytics (BDPA), an emerging technique that uses diagnostic tools and technological methods to obtain insights from big data in order to achieve improved performance, crisis management, enhanced innovation and increased productivity (Wamba et al., 2017).

Many previous studies have attempted to investigate the impact of Big Data and Predictive Analytics (BDPA) capability on organizational performance (Gupta and George, 2016). Braganza et al. (2017) conducted a study on the impact of BDPA on environmental sustainability and Jeble et al. (2018) studied the impact of BDPA on supply chain sustainability. Dubey et al. (2017) explored whether there is a significant effect of BDPA on improved social and environmental sustainability. Emmanouil and Nikolaos (2015) researched the role of big data analytics in crisis prevention. Moreover, Arslan et al. (2017) explained the impact of big data application on crisis management. After an extended review of these and related studies and literatures, a lack of research linking big data and predictive analytics with crisis management was discovered, particularly in the Arab context. Consequently, the current study focuses on the impact of BDPA on three crisis management phases, namely crisis preparedness, crisis response and crisis recovery. This study depends on the Resource-Based View (RBV) theory developed by Gupta and George (2016) to identify the dimensions of BDPA. They suggest that utilizing big data resources and the associated tools requires specialized capabilities if more informed business decisions and predictions are to be gained from big data. Consequently, Gupta and George (2016) determined five dimensions for BDPA consisting of (1) tangible resources, (2) technical skills, (3) management skills, (4) organizational learning and (5) data driven culture. Numerous Jordanian organizations, including the Greater Amman Municipality (GAM), utilize multiple big data techniques to collect information from widespread sources. Data collection and processing techniques used by GAM include an egovernment application, since 2005, as well as GIS, GPS, Mobile Application, Data Warehouse, Data Mining and numerous statistical techniques for data prediction (Mofleh et al., 2008). Despite the use of these techniques, GAM remains exposed to many crises, including natural threats such as excessive rain causing road closures, as well as administrative crises. The following main research question has been derived for the purpose of this study.

RQ1. Does BDPA capability have a significant impact on crisis management?

\section{Theoretical Background}

\section{Big Data and Predictive Analytics Capability}

Big data is defined as a complex set of structured and unstructured data that traditional technologies cannot process. It aims to detect hidden patterns, relies on models and is data science driven (Taylor-Sakyi, 2016). Chen et al. (2014) define big data as fulfilling four features: Data volume, data velocity, data variety and data veracity. Today, big data technology has become an important tool for improving organizational operations and decision making. Moreover, big data technology enables the early detection of many crises and disasters through interpretation of a combination of data from diverse sources (Orenga-Roglá and Chalmeta, 2016). The importance of big data is evident in the huge amount of data that is being produced, stored or bought from numerous sources by organizations around the world. Proper exploitation of this data contributes to the enhancement of an organization's status and its competitiveness by providing the company with ample information about the external environment in which they operate. Mawed and Al-Hajj (2017) argue that Big Data Analytics (BDA) through their software plays a critical role in enhancing the performance of administrative systems, facility management and crisis predictions. In the past, information, data and trends were complicated to assimilate. Currently, big data systems have made it much easier to visualize data, discover trends and expand algorithms to forecast when issues will arise (Creagh, 2016).

Despite the obvious benefits of collecting big data, many organizations have faced difficulties utilizing huge volumes of accumulated data, especially when information is extracted from multiple sources. Vast data volume often leads to organizational and formatting problems while attempting to process unstructured information. However, recent technological advancements have tackled many of these problems and big data techniques for overcoming volume and variety related issues have and continue to be developed (Orenga-Roglá and Chalmeta, 2016). Tools designed to remediate the challenge of data volume include the map reduce method, which permits parallel processing of large amounts of data. Likewise, metadata is a new method created to assist with processing data variety (Fertier et al., 2016). Nevertheless, many organizations continue to face difficulties fully utilizing large amounts of data, particularly in the face of crises, which requires fast integration, aggregation and visualization to account for rapid organizational decisions and emergency actions (Emmanouil and Nikolaos, 2015). Gathering, saving and analyzing enormous amounts of data calls for the establishment of new approaches to decision making that 
support effective data management (Watson, 2014). Many organizations continue to fail to reap the enormous benefits available from adopting big data and predictive analytics techniques due to lack of ability to utilize information fully. Especially lacking are trained professionals in required roles, as well as applicable technologies and processes and a corporate culture that encourages big data initiatives. Many organizations need to acquire further expertise in statistical techniques and analysis as well as in developing and implementing big data strategies. There is a need for IT specialists with the ability to choose the best big data techniques and algorithms, experience in dealing with data privacy issues and knowledge of integrating BDA activities with enterprise business processes (Mawed and Al-Hajj, 2017). Gupta and George (2016) identify some of the abilities needed to accumulate, integrate and deploy organizational big data into precise resources, which they define as BDPA capability. The following sections describe the five dimensions of BDPA, tangible resources, technical skills, management skills, organizational learning and data driven culture.

Tangible ressources: Ortha et al. (2015) define tangible resources as the material or substantial resources available to an organization, consisting of financial, manufactured and natural assets. Schriber and Löwstedt (2015) describe tangible resources as necessary antecedents of organizational capabilities. Reed (2005) likewise affirms tangible resources as important aspects of the implementation of organizational activities and as having an obvious impact on organizational routines. Jeble et al. (2018) state that tangible resources are the essential component needed to develop analytics solutions integrating capital, IT infrastructure, networks, communications, data sources and other resources. Hence, as big data technologies require analytics solutions of this complexity, tangible resources are a necessary investment for increasing big data analytics capability and allowing organizations to achieve their objectives in utilizing big data (Gupta and George, 2016).

Technical skills: Benefit from huge volumes of big data, whether homogeneous or heterogeneous, depends upon the expertise of big data analysts with necessary statistical analysis skills and full familiarity with the procedures and methodologies of interpreting big data, including machine learning and problem solving (Jeble et al., 2018). Davenport (2014) observed that many organizations are launching training programs to educate their staff accordingly, or else employing new personnel with expertise in dealing with data science and big data technology. Thus, related technical skills are a clear and intuitive prerequisite to effective utilization of big data.

Management skills: According to Ananthram and Nankervis (2013), management skills can be understood as the positive attributes and abilities needed by managers to achieve organization goals, including planning, communication, decision-making and delegation. Moreover, managers should have the ability to gather a team and align them in the direction of common goals. Appropriate management skills are vital for analytics projects since managers play a significant role in leadership and culture-building within a group. In addition, managers are responsible for overseeing communication and skill-building within a project, which is especially needed when dealing with the complexity of projects involving big data collection and analysis (Davenport, 2014). Therefore, effective organizational use of big data depends upon good management skills within that organization.

Organizational learning: In the face of accelerating environmental changes, it is necessary for organizations to increase their ability to quickly adapt and respond to situational shifts. In order for this to effectively occur organization staff must increase their knowledge of work procedures and learn from past mistakes and relevant experiences. Encouraging and incorporating employee knowledge will enhance organizational learning and lead to competitive advantages. Gupta and George (2016) argue that organizational learning plays an important role in enhancing big data capabilities since it leads to more effective use of new techniques.

Data driven culture: The culture of an organization, which is developed over the organization's lifespan, plays a major role in the adoption of IT systems (Ozigbo, 2013). Many previous studies, such as that conducted by Lavalle et al. (2011), have confirmed that the success of big data projects and organizational ability to analyze them and make appropriate decisions requires building a data-driven culture. According to McAfee and Brynjolfsson (2012) data-driven culture is a key resource contributing to big data capabilities.

\section{Crisis Management}

Coombs (2011) defines crisis as an unpredictable event that threatens the expectations of stakeholders and can seriously affect the performance of an organization and generate adverse consequences. Dufort (2007) indicates that crises in organizations are inevitable and forecasting and planning for their management is one of the most important responsibilities of managing organizations. Crisis management is an attempt to control events and damages throughout an emergency, including monitoring the course of the crisis, maintaining control of its development and reducing its risks (Yamamoto and Skerogu, 2011). Crisis management is one of the most important functions of management. It demands planning and rapid response to situations before their progression, which can be unpredictable and may lead to serious damages (Almansoori and Habtoor, 2018). Information 
technology has become one of the key tools in effective crisis management. ICTs can be used to support the work of international communities in crisis areas, to improve the exchange of information in crisis situations in a more secure and confidential manner, in addition to enhance information quality (Rinkineva, 2004). Crisis phases have been classified by researchers with different criteria. Most classifications include three stages, namely crisis preparedness, crisis response and crisis recovery. Crisis preparedness refers to preventive activities that seek to reduce the occurrence of crises. In addition to planning methods of avoiding emergencies, this phase includes training crises management teams to deal with disaster events. Crisis response focuses on the speed and effectiveness of the initial response to emergency situations, including containing damages and preventing calamities from spreading to other parts of the organization. This phase of crisis management may take different forms based on the nature of the incident. The final stage of crisis management is crisis recovery, which includes restoring activity to the tangible and intangible assets that were lost during the crisis, learning from the situation and setting the necessary controls and standards to prevent recurrence (Alexander, 2015; Emmanouil and Nikolaos, 2015).

\section{Research Model and Hypotheses}

Big data and the ability to analyze it are critical topics in the modern era, as is crisis management. These topics present challenges, as well as enormous potential benefits, to organizations around the world. The literature and research related to these themes were reviewed and found to be insufficient in proportion to their importance. After review, the study model was constructed as shown in Fig. 1.

According to the literature review, big data and predictive analytics capability cannot be separate topics while playing a vital role in crisis management, as shown in the study model above. In the following sections, the role of big data and predictive analytics capability will be explained in each phases of crisis management and the hypotheses will be proposed.

\section{Crisis Preparedness}

Accurate and timely information helps organizations plan appropriate processes for crisis management and reduce the chance of reoccurrence. Planning for crisis consequences is difficult and requires complex models and different scenarios due to multiple variables and unpredictability associated with crises. Consequently, big data and predictive analytics capability can be hugely instrumental in improving scenario modeling and crisis forecasting (Pu and Kitsuregawa, 2013). Rahman et al. (2017) argue that big data may contribute to the detection of early warning signs for various crises by integrating and coordinating data from multiple sources. Big data analysis can help forecasting via satellite and atmospheric data joined with statistical analysis (Jongman et al., 2015). Analysis of big data helps prepare for crisis management by identifying risks and providing a strategic approach to managers for dealing with crises proactively and preemptively compiling necessary resources. GIS can be used to formulate projects that help plan for disaster preparedness and disaster relief activities (Emmanouil and Nikolaos, 2015). Qadir et al. (2016) confirms that big data analytics can be useful in the crisis preparedness stage. Since crises are multifarious, have inconsistent effects and can move at unstable speeds, harnessing the potential of big data technology can allow organizational capacity to manage crises to be significantly enhanced. Big data amassed from social media and mobile networks presents an excellent opportunity to gain valuable insights from consumers. This information can allow organizations to measure levels of client satisfaction and forecast potential future crises. Based on the above discussion, the following hypothesis is proposed.

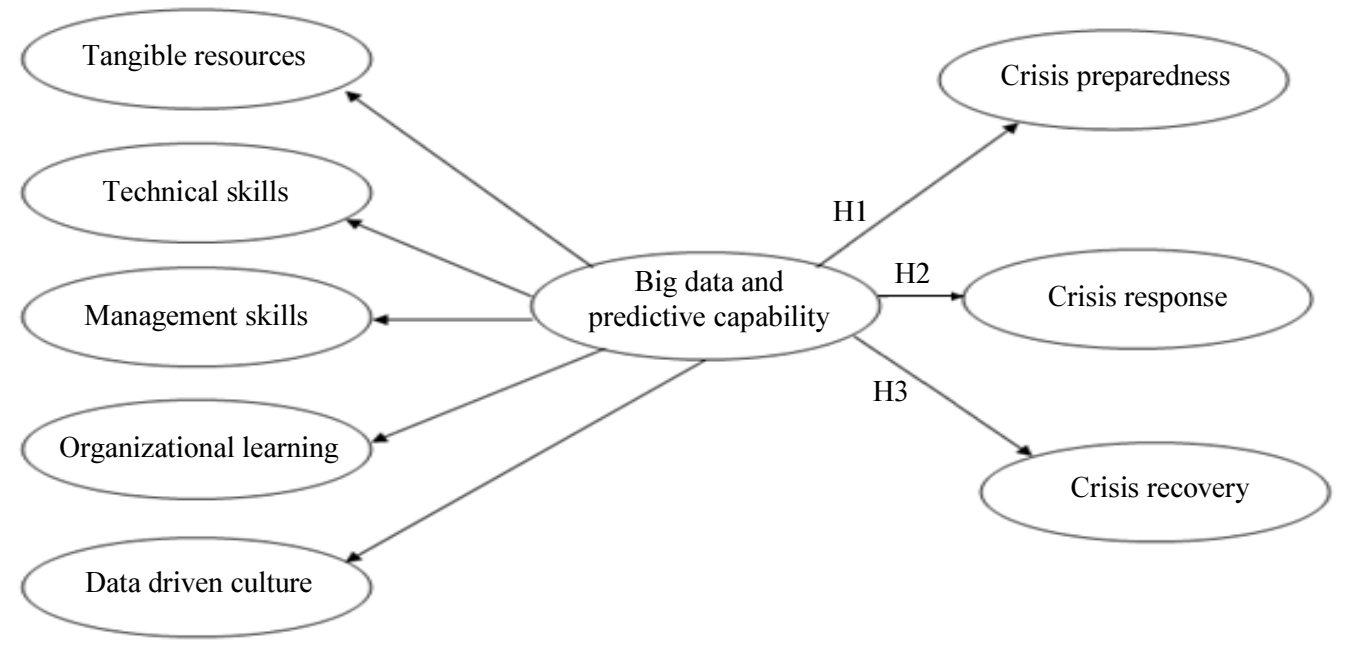

Fig. 1: Study model 
H1: Big data and predictive analytics capability has a significant impact on Crisis Preparedness

\section{Crisis Response}

During crises, organizations experience a constant stream of incoming data that requires effective processing to transform it into useful information (Gibson et al., 2014). Qadir et al. (2016) argue that big data from mobile phones and social media throughout a crisis can help management to respond effectively to the situation. Arslan et al. (2017) assert that big data technology and predictive analytics capability are instrumental in crisis management, provide examples such as RFID systems which assist in environmental data generation and tracking of people. Big data can help identify areas where citizens need assistance and indicate where to assign resources through the GIS system. $\mathrm{Pu}$ and Kitsuregawa (2013) describe three regions where big data can enhance management crisis response. These are detecting the dangerous area, real time situation analysis and identification of the most effective response based on historical cases. Therefore, the following hypothesis is proposed.

$\mathrm{H} 2$ : Big data and predictive analytics capability has a significant impact on Crisis Response

\section{Crisis Recovery}

Big data aids crisis recovery procedures through providing useful information about volunteer directions and logistics during and after the crisis. The recovery stage may be short or long-term, ranging from weeks to years, as time needed for regaining organizational health and financial recovery can vary. Moreover, the crisis recovery phase requires extended periods of planning and ample resource provisions in order to restore the organization to its original position (Gibson et al., 2014). Big data and analytical predictive capability enhances the success of recovery activities through utilizing data compiled during the crisis preparedness phase (Arslan et al., 2017). Emmanouil and Nikolaos (2015) argue that GIS and social media can deliver accurate information regarding what has been damaged, where and the number of individuals or organizations affected. This kind of information is extremely useful to the recovery process. Based on the above explanation, we pose the following hypothesis.

H3: Big data and predictive analytics capability has a significant impact on Crisis Recovery

\section{Research Methodology}

\section{The Measurement}

After reviewing the research and literature related to the subject of the study and conducting visits and interviews in the Greater Amman Municipality (GAM), the dimensions of the study were formulated as shown in Table 1.

\section{Instrument Development}

Many relevant studies were used to organize the study questions, as demonstrated in Table 2. The survey used to gather information for the study contains 32 questions. The big data and predictive capability construct was adopted from (Jeble et al., 2018; Dubey et al., 2017; Gupta and George, 2016), while the crisis management construct was developed from (Arslan et al., 2017; Burns, 2012; Sawalha et al., 2013). A five-point Likert-scale was used for the study. Seven IT professionals, management information systems and business management participated in judging the survey questions and gave us their comments and feedback for its improvement.

\section{Sampling and Questionnaire Distribution}

The purposive sample method was used to select study sample individuals, as it was deemed best suited to achieving the study objective. 140 questionnaires were distributed to senior management staff, 128 of which were deemed valid for analysis.

\section{Data Analysis and Results}

Structural Equation Modeling was applied through PLS. 2 software to examine the collected data. The result of factor loading for each item in the study model was appropriate and exceeded the cut value $(0.50)$ as shown in Fig. 2. Consistency and convergent validity were confirmed based on Cronbach's alpha, Rho A, Composite Reliability (CR) and (AVE), as shown in Table 2.

As reflected in the values in the above table, all constructs achieved adequate value beyond the necessary threshold.

\section{Assessing the Structural Model and Testing Research Hypotheses}

In this study, the PLS algorithm was used to evaluate the structural model and discover the significance of the paths and the predictive power of the model as shown in Fig. 2.

As shown in Table 3, three paths were significant, indicating that their three associated hypotheses are supported (H1, H2 and $\mathrm{H} 3)$. The results depict that big data and predictive capability has a significant impact on Crisis Preparedness (the path coefficients of relationships is 0.811 , with $t$ value $=16.156$ and $P$ value $=0.000$, where the $\mathrm{R}^{2}$ value was 0.658 ), meaning that big data and predictive capability explained $0.65 \%$ of Crisis Preparedness. Big data and predictive capability also demonstrated a significant impact on Crisis Response (the path coefficients of relationships is 0.455 , with $t$ value $=3.820, P$ value $=0.000$, where the $\mathrm{R}^{2}$ value 
was 0.207), meaning that big data and predictive capability explained $0.20 \%$ of Crisis Response. Finally, big data and predictive capability has a significant impact on Crisis Recovery (the path coefficients of relationships is 0.546 , with $t$ value $=3.715, P$ value $=$ 0.000 , where the $\mathrm{R}^{2}$ value was 0.298 ), which means that big data and predictive capability explained $0.29 \%$ of Crisis Recovery.

Table 1: Study instruments

\begin{tabular}{|c|c|c|}
\hline Construct & Code & Measurement items \\
\hline \multirow{2}{*}{\multicolumn{3}{|c|}{$\begin{array}{l}\text { Big data and predictive capability } \\
\text { Tangible Resources (TR) }\end{array}$}} \\
\hline & & \\
\hline & TR1 & $\begin{array}{l}\text { GAM staff has right of entry to huge, unstructured, quick-moving data for inspection } \\
\text { and analysis. }\end{array}$ \\
\hline & TR2 & $\begin{array}{l}\text { Data is collected from multiple internal sources within GAM and integrated into a } \\
\text { unified warehouse. }\end{array}$ \\
\hline & TR3 & $\begin{array}{l}\text { Internal data is integrated with data from external sources to facilitate analysis and } \\
\text { utilization in crisis management. }\end{array}$ \\
\hline \multirow[t]{4}{*}{ Technical Skills (TS) } & ST1 & GAM provides its staff with the necessary training to handle big data. \\
\hline & ST2 & $\begin{array}{l}\text { GAM is interested in hiring staff with sufficient skills to utilize big data and make } \\
\text { predictive analyses. }\end{array}$ \\
\hline & ST3 & $\begin{array}{l}\text { GAM staff members have and use analytical and predictive capabilities through big } \\
\text { data to accomplish their work and address emergencies. }\end{array}$ \\
\hline & ST4 & $\begin{array}{l}\text { Big data and predictive analytics staff members in GAM are well qualified and } \\
\text { appropriately educated. }\end{array}$ \\
\hline \multirow[t]{4}{*}{ Management Skills(MS) } & MS1 & $\begin{array}{l}\text { Big data and predictive analytics managers in GAM recognize sustainable business } \\
\text { improvement needs of other functional managers, suppliers and customers. }\end{array}$ \\
\hline & MS2 & $\begin{array}{l}\text { Big data and predictive analytics managers in GAM use their knowledge to support } \\
\text { other stakeholders. }\end{array}$ \\
\hline & MS3 & $\begin{array}{l}\text { Big data and predictive analytics managers in GAM have enough skills to explore } \\
\text { opportunities that big data might bring to their jobs. }\end{array}$ \\
\hline & MS4 & $\begin{array}{l}\text { Big data and predictive analytics managers in GAM are able to recognize and } \\
\text { estimate big data outcomes. }\end{array}$ \\
\hline \multirow[t]{3}{*}{ Organizational Learning (OL) } & OL1 & GAM staff can easily investigate new and related knowledge. \\
\hline & OL2 & GAM staff can attain new and applicable knowledge related to their jobs. \\
\hline & OL3 & GAM staff can understand new and related knowledge attained from big data. \\
\hline \multirow[t]{3}{*}{ Data Driven Culture (DDC) } & DDC1 & GAM staff looks to data as a substantial asset. \\
\hline & DDC2 & GAM staff depends on big data and predictive analytics in decision making \\
\hline & DDC3 & $\begin{array}{l}\text { GAM staff uses big data and predictive analytics in decision making more than } \\
\text { intuition or instinct. }\end{array}$ \\
\hline \multicolumn{3}{|l|}{ Crisis management } \\
\hline \multirow[t]{5}{*}{ Crisis Preparedness (CP) } & CP1 & $\begin{array}{l}\text { The statuses of various areas of GAM are continuously studied to detect early crisis } \\
\text { warning signals. }\end{array}$ \\
\hline & $\mathrm{CP} 2$ & $\begin{array}{l}\text { GAM staff shows an interest in collecting and detecting danger signs that may be } \\
\text { predictors of crises. }\end{array}$ \\
\hline & CP3 & Any detection of warning signals for potential crises is quickly handled by GAM. \\
\hline & CP4 & GAM has pre-prepared solutions (scenarios) to respond to predictable crises. \\
\hline & CP5 & All errors and complaints are taken seriously by GAM staff. \\
\hline \multirow[t]{5}{*}{ Crisis Response (CR) } & CR1 & $\begin{array}{l}\text { Professional and technical methods of dealing with crises and containing damages } \\
\text { are provided to GAM staff. }\end{array}$ \\
\hline & CR2 & $\begin{array}{l}\text { During a crisis, GAM has alternative communication methods in case the primary } \\
\text { method crashes. }\end{array}$ \\
\hline & CR3 & Multiple departments within GAM are involved in implementing crisis management plans. \\
\hline & CR4 & Communications and operations are kept confidential by GAM during a crisis. \\
\hline & CR5 & Tasks and powers are quickly allocated within GAM to contain the damages of a crisis. \\
\hline \multirow[t]{5}{*}{ Crisis Recovery (CRR) } & CRR1 & All information about crises is stored by GAM for the purpose of future utilization. \\
\hline & CRR2 & $\begin{array}{l}\text { GAM takes all recommendations and solutions proposed to resolve previous crises } \\
\text { into account. }\end{array}$ \\
\hline & CRR3 & $\begin{array}{l}\text { Past crisis management plans and programs are evaluated by GAM with the } \\
\text { intention of developing and improving them in order to address future crises better. }\end{array}$ \\
\hline & CRR4 & The necessary procedures are taken by GAM to continue normal activity without delay. \\
\hline & CRR5 & $\begin{array}{l}\text { Multiple forms of communication are used by GAM to inform citizens about the } \\
\text { consequences of a crisis and how to deal with it. }\end{array}$ \\
\hline
\end{tabular}


Table 2: Validity and reliability estimates of the constructs

\begin{tabular}{|c|c|c|c|c|}
\hline Construct & AVE & Composite reliability & Cronbach's alpha & Rho A \\
\hline Tangible Resources (TR) & 0.605 & 0.817 & 0.695 & 0.731 \\
\hline Technical Skills (TS) & 0.631 & 0.871 & 0.801 & 0.802 \\
\hline Management Skills (MS) & 0.654 & 0.883 & 0.822 & 0.820 \\
\hline Organizational Learning (OL) & 0.736 & 0.893 & 0.821 & 0.830 \\
\hline Data Driven Culture (DDC) & 0.747 & 0.899 & 0.831 & 0.831 \\
\hline Big Data and Predictive Capability & 0.502 & 0.938 & 0.921 & 0.938 \\
\hline Crisis Preparedness $(\mathrm{CP})$ & 0.609 & 0.885 & 0.835 & 0.855 \\
\hline Crisis Response (CR) & 0.718 & 0.927 & 0.902 & 0.915 \\
\hline Crisis Recovery (CRR) & 0.651 & 0.903 & 0.866 & 0.869 \\
\hline
\end{tabular}

Table 3: The path coefficient and hypotheses results

\begin{tabular}{lllll}
\hline H\# & Path coefficient $(\beta)$ & T statistics & $P$ value & The results \\
\hline H1 & $0.811^{* *}$ & 16.156 & 0.000 & Supported \\
H2 & $0.455^{* *}$ & 03.820 & 0.000 & Supported \\
H3 & $0.546^{* *}$ & 03.715 & 0.000 & Supported \\
\hline$* * P<0.05$ & & &
\end{tabular}

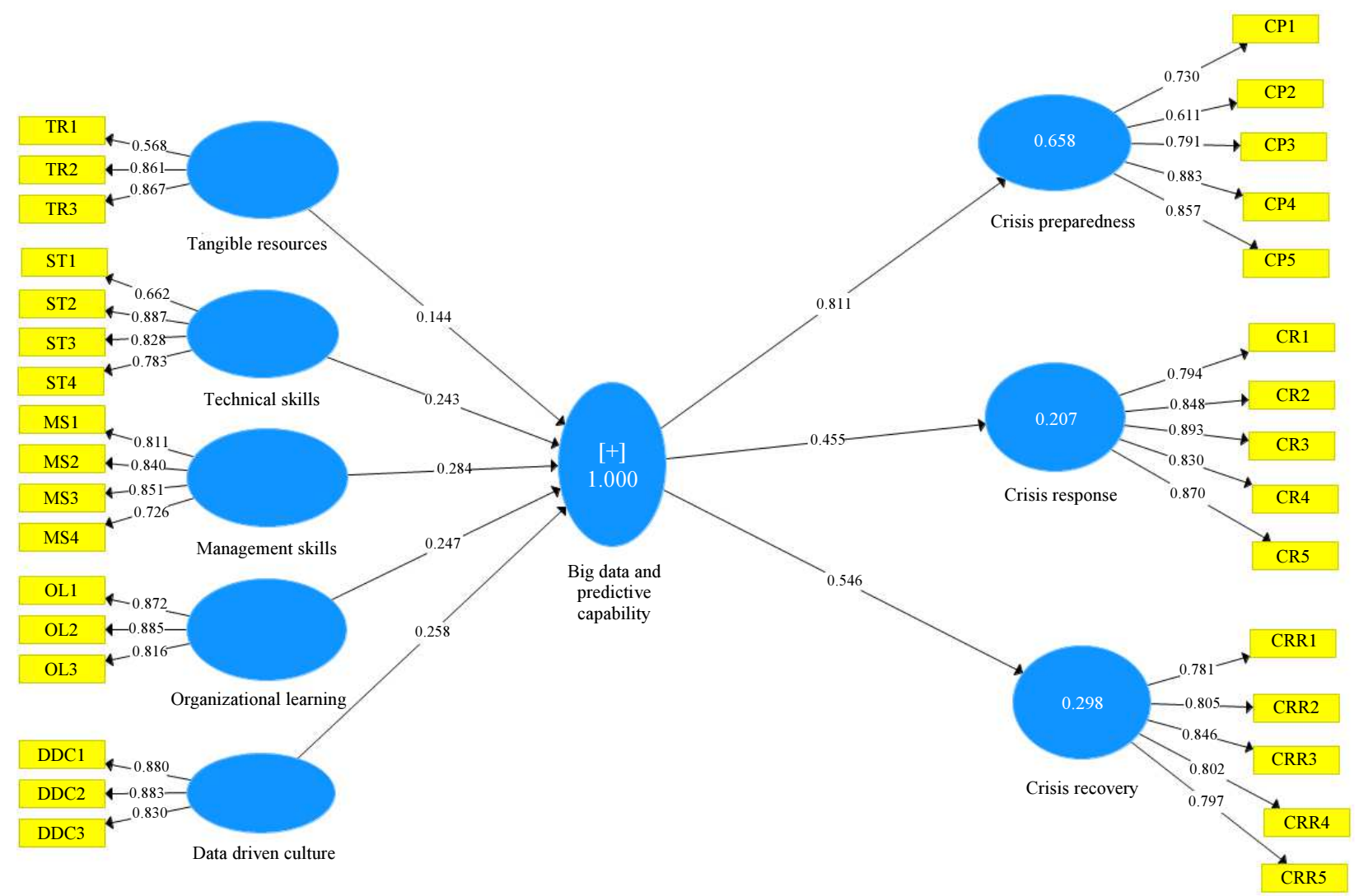

Fig. 2: Tested research model

\section{Discussion, Conclusion and Implication}

Big data, more precisely Big Data and Predictive Analytics (BDPA), is a new trend in the organizational environment of Jordan, especially in the Greater Amman Municipality. In the Jordanian context, there remains little profound understanding of BDPA and the specific capabilities needed to better utilize these new technologies. The purpose of this study is to offer an understanding of big data and predictive analytics capability and explain its impact on three crisis management stages. Moreover, the current study investigates the impact of big data and predictive analytics capability, in all five dimensions of tangible 
resources, technical skills, management skills, organizational learning and data driven culture, on the three crisis management stages of preparedness, response and recovery. The study results show that GAM has medium levels of BDPA capability. Furthermore, the study findings demonstrate that BDPA capability has a significant impact on crisis management.

Big data and predictive analytics capability demonstrated an especially significant impact on crisis preparedness, with the value of path coefficient $(\beta)$ at 0.811 and $P$ value $=0.000$, asserting the importance of BDPA capability in this area. Big data sources such as mobile applications, data warehouses and social networks can support organizations by providing large volumes of information useful in crisis avoidance and preparation for emergency events. Organizations can monitor the trends of their stakeholders via social media outlets such as Facebook, Twitter and blogs before or during times of crisis. Al-Ma'aitah (2019) argues that web 2.0 technologies can help organizations gather data about services to meet the needs and expectations of citizens. These results agree with the research of (Arslan et al., 2017; Emmanouil and Nikolaos, 2015; Rahman et al., 2017). Accordingly, organizations are recommended to build BDPA capability to aid them in crisis prevention.

The study also found that big data and predictive analytics capability has a significant impact on crisis response, with the values of path coefficient $(\beta)$ at 0.455 and $P$ value $=0.000$. These results confirm the importance of BDPA capability on organizations' ability to respond to crisis. Big data tools such as GIS and data mining can provide the answers to the most pressing questions facing management when attempting to respond effectively to crises as well as assisting in analysis processing. BDPA can help address technical challenges linked with processing big data, making it an important area for organizations to consider. These results concurred with (Gibson et al., 2014; Arslan et al., 2017; Emmanouil and Nikolaos, 2015; Rahman et al., 2017; Qadir et al., 2016).

The final result of the study model testing shows that big data and predictive analytics capability has a significant impact on crisis recovery. The values of path coefficient $(\beta)$ was 0.546 with $P$ value $=0.000$. This is in part explained by the fact that big data tools such as social media can help to disseminate information over a number of channels to the stakeholders. Big data storages and data bases help effectively supplement crisis recovery activities. Organizations are recommended to enhance their BDPA capabilities in order to prepare plans and programs needed to perform crisis recovery. This result matched studies by (Emmanouil and Nikolaos, 2015; Bekhor et al., 2015; Rahman et al., 2017).
Analyzing the study findings, we note that the most significant impact of BDPA was on crisis preparedness, followed by crisis response and finally crisis recovery. Based on the above discussion, we emphasis that organizations should enhance their tangible resources infrastructure related to processing big data, such as machine learning algorithms, python software, simulations and regression analysis for analyzing huge volumes of data. In addition, organizations should improve their technical skills by providing their staff with the necessary training to handle big data analysis. Furthermore, management skills should be improved to more effectively explore opportunities that big data might support and to adopt big data projects through enhancing organizational learning and building a strong data driven culture. Organizations should consider big data technologies to be enabling technologies that enhance crisis management and inform decision making processes. Finally, organizations should highlight the importance of big data and predictive analytics capability in crisis management to their staff and disseminate a data driven culture throughout all levels of their organization.

\section{Acknowledgement}

I would like to acknowledge the editors of this journal and the reviewers for reviewing and evaluating this manuscript and providing fitting feedbacks

\section{Ethics}

This article is original and contains unpublished material.

\section{References}

Alexander, D.E., 2015. Disaster and Emergency Planning for Preparedness, Response and Recovery. In: Oxford Research Encyclopedia of Natural Hazard Science, Oxford University Press, pp: 1-20.

Ananthram, S. and A. Nankervis, 2013. Strategic agility and the role of $\mathrm{HR}$ as a strategic business partner: An Indian perspective. Asia Pacific J. Human Resources, 51: 454-470.

Al-Ma'aitah, M., 2019. Drivers of e-government citizen satisfaction and adoption: The case of Jordan. Int. J. E-Bus. Res.

Almansoori, M. and N. Habtoor, 2018. The role of social media on crisis preparedness and responses. Int. J. Scientific Eng. Res., 9: 1347-1353.

Arslan, M., A. Roxin, C. Cruz and D. Ginhac, 2017. A review on applications of big data for disaster management. Proceedings of the 13th International Conference on Signal Image Technology Internet Based Systems, Dec. 4-7, IEEE Xplore Press, Jaipur, India. DOI: 10.1109/SITIS.2017.67 
Bekhor, S., Y. Doytsher, S. Cohen and Y. Kanza, 2015. A personalized geo social app for surviving an earthquake. Proceedings of the 1st ACM SIGSPATIAL International Workshop on the Use of GIS in Emergency Management, Bellevue, (EMB' 15), WA, USA. DOI: $10.1145 / 2835596.2835616$

Besaleva, I. and A.C. Weaver, 2016. Applications of social networks and crowd sourcing for disaster management improvement. Computer, 49: 47-53. DOI: 10.1109/MC.2016.133

Braganza, A., L. Brooks, D. Nepelski, M. Ali and R. Moro, 2017. Resource management in big data initiatives: Processes and dynamic capabilities. J. Bus. Res., 70: 328-337.

DOI: $10.1016 / \mathrm{j}$.jbusres.2016.08.006

Burns, K.F., 2012. Assessing and exploring strategic crisis management planning in Michigan small businesses.

Chen, M., S. Mao and Y. Liu, 2014. Big data: A survey. Mobile Netw. Applic., 19: 171-209. DOI: $10.1007 / \mathrm{s} 11036-013-0489-0$

Coombs, W.T., 2011. Protecting organization reputations during a crisis: The development and application of situational crisis communication theory. Corporate Reputat. Rev., 10: 163-176. DOI: $10.1057 /$ palgrave.crr. 1550049

Creagh, B., 2016. Crown Melbourne capitalizes on big data to enhance FM. FM Online Magazine.

Davenport, T., 2014. How strategists use "big data" to support internal business decisions, discovery and production. Strategy Leadership, 42: 45-50.

Dubey, R., A. Gunasekaran, S. Childe, D. Roubaud and Z. Luo et al., 2017. Can big data and predictive analytics improve social and environmental sustainability? Technol. Forecast. Soc. Change, 144: 534-545. DOI: 10.1016/j.techfore.2017.06.020

Dufort, C., 2007. Is crisis management (Only) a management of exceptions? J. Contingencies Crisis Manage., 15: 105-114.

DOI: $10.1111 / \mathrm{j} .1468-5973.2007 .00507 . \mathrm{x}$

Emmanouil, D. and D. Nikolaos, 2105. Big data analytics in prevention, preparedness, response and recovery in crisis and disaster Management. In: Recent Advances in Computer Science, ISBN-13: 978-1-61804-320-7 pp: 476-482.

Fertier, A., A. Montarnal, A.M. Barthe-Delanoë, S. Truptil and F. Bénaben, 2016. Adoption of big data in crisis management toward a better support in decisionmaking. Proceedings of the International ISCRAM Conference, (IIC' 16), Rio de Janeiro, Brazil.

Gibson, H., S. Andrews, K. Domdouzis, L. Hirsh and B. Akhgar, 2014. Combining big social media data and FCA for crisis response. Proceedings of the 7 th International Conference on Utility and Cloud Computing, Dec. 8-11, IEEE Xplore Press, London, UK. DOI: 10.1109/UCC.2014.112
Gupta, M. and J.F. George, 2016. Toward the development of a big data analytics capability. Inform. Manage., 53: 1049-1064.

Jeble, S., R. Dubey, S. Childe, T. Papadopoulos and D. Roubaud et al., 2018. Impact of big data and predictive analytics capability on supply chain sustainability. Int. J. Logist. Manage., 29: 513-538. DOI: 10.1108/IJLM-05-2017-0134

Jongman, B., J. Wagemaker, B.R. Romero and E.C. de Perez, 2015. Early flood detection for rapid humanitarian response: Harnessing near real-time satellite and Twitter signals. ISPRS Int. J. Geo. Inform., 4: 2246-2266.

DOI: $10.3390 /$ ijgi4042246

LaValle, S., E. Lesser, R. Shockley, M.S. Hopkins and N. Kruschwitz, 2011. Big data, analytics and the path from insights to value. MIT Sloan Manage. Rev., 52: 21-32.

Lu, Y. and K. Ramamurthy, 2011. Understanding the link between information technology capability and organizational agility: An empirical examination. MIS Quarterly, 35: 931-954. DOI: $10.2307 / 41409967$

Mawed, M. and A. Al-Hajj, 2017. Using big data to improve the performance management: A case study from the UAEFM industry. Facilities, 35: 746-765. DOI: $10.1108 / \mathrm{F}-01-2016-0006$

McAfee, A. and E. Brynjolfsson, 2012. Big data: The management revolution. Harvard Business Review.

Mitroff, I., P. Shrivastava and E. Firdaus, 1987. Effective crisis management. Acad. Manage. Executive, 1: 283-292. DOI: $10.5465 /$ ame.1987.4275639

Mofleh, S., M. Wanous and P. Strachan, 2008. Developing countries and ICT Initiatives: Lessons learnt from Jordan's experience. Electro. J. Inform. Syst. Dev. Country, 34: 1-17. DOI: $10.1002 / j .1681-4835.2008 . t b 00236 . x$

Orenga-Roglá, S. and R. Chalmeta, 2016. Social customer relationship management: Taking advantage of Web 2.0 and big data technologies. Springer Plus, 5: 2-17.

DOI: $10.1186 / \mathrm{s} 40064-016-3128-y$

Ortha, R., R. Scheumannb, M. Galeitzkec, K. Wolfb and H. Kohla et al., 2015. Sustainable corporate development measured by intangible and tangible resources as well as targeted by safeguard subjects. Proc. CIRP, 26: 630-634. DOI: $10.1016 /$ j.procir.2014.07.113

Ozigbo, N.C., 2013. Impact of organizational culture and technology on firm performance in the service sector. Commun. IIMA.

$\mathrm{Pu}, \mathrm{C}$. and M. Kitsuregawa, 2013. Big data and disaster management a report from the JST/NSF joint workshop. Georgia Institute of Technology, CERCS. 
Qadir, J., A. Ali, R. Rasool and A. Zwitter, 2016. Crisis analytics: Big data-driven crisis response. J. Int. Human. Act., 1: 12-12. DOI: 10.1186/s41018-016-0013-9

Rahman, S., E. Zannat and L. Di, 2017. The role of big data in disaster management. Proceedings of the International Conference on Disaster Risk Mitigation, (DRM' 17), Dhaka, Bangladesh, pp: 23-24.

Reed, M., 2005. Reflections on the realist turn in organization and management studies. J. Manage. Stud., 42: 1621-1644.

DOI: $10.1111 / \mathrm{j} .1467-6486.2005 .00559 . \mathrm{x}$

Rinkineva, K., 2004. The role of information technology in crisis management. Proceedings of the 14th European Information Network on International Relations and Area Studies, (RAS' 04).

Sawalha, H., E. Jraisat and K. Al-Qudah, 2013. Crisis and disaster management in Jordanian hotels: Practices and cultural considerations. Disaster Prevent. Manage., 22: 210-228. DOI: $10.1108 /$ DPM-09-2012-0101

Schriber, S. and J. Löwstedt, 2015. Tangible resources and the development of organizational capabilities. Scandinavian J. Manage., 31: 54-68. DOI: $10.1016 /$ j.scaman.2014.05.003
Taylor-Sakyi, K., 2016. Big data: Understanding big data. Wamba, S.F., A. Gunasekaran, S. Akter, S.J.F. Ren and R. Dubey et al., 2017. Big data analytics and firm performance: Effects of dynamic capabilities. J. Bus. Res., 70: 356-365. DOI: 10.1016/j.jbusres.2016.08.009

Wamba, S.F., S. Akter, A. Edwards, G. Chopin and D. Gnanzou, 2015. How 'big data' can make big impact: Findings from a systematic review and a longitudinal case study. Int. J. Prod. Econ., 165: 234-246. DOI: 10.1016/j.ijpe.2014.12.031

Watson, H., 2014. Tutorial: Big data analytics: Concepts, technologies and applications. Commun. Assoc. Inform. Syst., 34: 1248-1265. DOI: $10.17705 / 1 C A I S .03465$

Yamamoto, G. and Ö. Skerogu, 2011. Crisis management in the Turkish leather industry. African J. Bus. Manage., 5: 3212-3219.

Zeitun, R. and H. Benjelloun, 2013. The efficiency of banks and the financial crisis in a developing economy: The case of Jordan. J. Finance Account. Manage., 4: 1-20. 